\title{
Oculomotor nerve paresis in a case of scleritis
}

\author{
M. S. DAVIES AND A. G. KARSERAS \\ Moorfields Eye Hospital, London
}

\section{Case report}

A 59-year-old white woman attended the casualty department on September 23,1968 , with a $\dot{c}$ history of increasing pain and swelling of the left side of the face, and redness of the left eye during $\vec{\oplus}$ the previous week. She had suffered from "rheumatism" in her arms for many years, but otherwise had no serious or significant illness until 3 years previously when she had been found to be hypertensive. Since that time she had been treated with Methyl Dopa $250 \mathrm{mg}$. twice daily.

\section{Examination}

The right eye was normal in all respects. The left eye showed some swelling of the lids and peri- $\stackrel{\vec{\theta}}{-}$ orbital tissues. The sclera was injected, with overlying chemosis, and the eye was very tender to palpation. There was no staining of the corneal epithelium, the stroma was clear, and there were no inflammatory signs in the anterior chamber. The pupils were equal and showed normal reactions. The lens, vitreous, and posterior pole showed no abnormality, and the corrected visual acuity was normal.

Diagnosis

The case was diagnosed as one of scleritis.

\section{Treatment}

Topical steroids (Predsol-N) were administered.

Course

When she was next seen 2 days later the pain in the left eye was more intense, and the following day there was an increased oedema of the lids which suggested the possibility of an orbital infection. Treatment was changed to Penicillin-V $500 \mathrm{mg}$. four times a day.

The next day, 4 days after she was first seen, the pain was much worse. The left eye was extremely of tender and the sclera intensely injected, and visual acuity had dropped to 6/18. The eye was $\tilde{D}$ slightly proptosed (exophthalmometry $15 \mathrm{~mm}$. right, $19 \mathrm{~mm}$. left) and there was limitation of $\mathrm{N}^{N}$ adduction and elevation. The most striking feature was a complete ptosis; when the lid was raised the pupil was found to be dilated with no reaction to light or convergence, and the patient complained of double vision. The anterior chamber was shallow and showed a faint flare. The vitreous $\Phi$ was clear and the intraocular pressure was normal. Fundus examination revealed a peripheral choroidal detachment on the temporal side extending from 2 to 4 o'clock.

She was admitted to hospital where a general physical and neurological examination failed to reveal any other abnormality. 


\section{Laboratory Investigations}

Full peripheral blood count,

Erythrocyte sedimentation rate.

Latex test for rheumatoid factor.

Serum proteins.

Immunofluorescence test for antinuclear factor.

Examination for lupus erythematosus cells

Wassermann reaction and Kahn test

Radiological examination of skull, orbit, nasal sinuses, and chest.

Ultra-sound examination of eyeball and orbit.

The results of these investigations were normal except for some evidence of pleural thickening on the chest film, and elevation of the ESR to $32 \mathrm{~mm}$./ $1 \mathrm{st} \mathrm{hr}$.

\section{Further diagnosis and treatment}

It was considered that the original diagnosis of scleritis was correct, and treatment was recommenced on the day of admission to hospital with topical steroids; systemic steroids were witheld in view of the evidence of previous pulmonary inflammation on the chest $X$-ray. The penicillin which had been started the day before was discontinued, and no other therapy was given apart from analgesics.

For 48 hours the symptoms and signs remained unchanged, but the following day there was a slight subjective improvement and objectively the external ocular movements became less restricted. The pupil was less dilated and showed a sluggish response to light. Over the next few days the signs gradually regressed, except for the choroidal detachment which extended to involve the entire periphery of the fundus. Six days after admission it was possible to record the external ocular movements on a Hess chart, and 2 days later the ptosis and proptosis were only slight. The visual acuity improved to a normal level, and the pupil reacted briskly although it remained slightly larger than on the right. The annular choroidal detachment persisted, but 2 weeks after the first examination it showed signs of resolution, and the patient was discharged from hospital 3 days later.

\section{Follow-up}

When repeated 2 weeks and I month later the ESR was found to be normal.

\section{Comment}

It is usual to regard scleritis as occurring in two main forms, anterior and posterior. Anterior scleritis usually occurs in young people, and is more common in females than in males. In the most severe cases an annular scleritis may occur in association with extreme pain. Complications are common, including a secondary sclerosing keratitis and an intense uveitis which may predispose to the development of an exudative retinal detachment. An anterior choroidal detachment may occur infrequently. Posterior scleritis is characterized by oedema of the lids, proptosis, and impaired painful mobility of the globe.

There is marked chemosis, but the cornea and anterior segment remain normal, and the vision may be unimpaired except for the awareness of diplopia. There is an inflammatory infiltration within the extrinsic ocular muscles and also within the other orbital tissues, including even the lacrimal gland. Other intraocular complications have been described; vitreous opacities, choroiditis, retrobulbar neuritis, and exudative retinal detachment (Sears, 1964).

It is evident that this case shows some of the features of both anterior and posterior scleritis, but the main interest lies in the presence of the third cranial nerve paresis, which 
has not been recorded before in this condition. The latter was indicated by the complete ptosis, the fixed dilated pupil, and reduced elevation and adduction.

\section{Summary}

A case of scleritis is presented which was complicated by a third cranial nerve paresis: this complication has not been reported before. An annular choroidal detachment was also present. Full investigation failed to reveal the aetiology of the scleritis, which ran a benign course without recourse to systemic steroids.

Our thanks are due to Mr. K. C. Wybar for permission to publish details of his case and for his assistance in the preparation of this paper.

\section{Reference}

SEARs, м. L. (1964) Amer. J. Ophthal, 58, 764 